\title{
MedienPädagogik
}

Zeitschrift für Theorie und Praxis der Medienbildung

\section{Editorial: Zurück in die Zukunft der Medienpädagogik «Subjekt», «Bildung» und «Medien`Kritik» im Lichte | im Schatten digitaler Daten}

Valentin Dander

Vorwort

\section{Zur Genese}

Die vorliegende Dissertation dokumentiert einen Forschungsprozess, der alles andere als geradlinig bezeichnet werden kann. Beginnend mit April 2012 wurde mehr oder minder kontinuierlich daran gearbeitet. Den Startschuss markierte ein Antrag um ein Dissertationsstipendium aus der Nachwuchsförderung der Leopold-FranzensUniversität Innsbruck, für welches ein Forschungsvorhaben über das neu online gestellte Open Government Data Portal des Landes Tirol ersonnen wurde. Nicht nur lief nach einem Jahr die finanzielle Förderung aus, worauf keine Anschlussfinanzierung folgte, auch erwies sich das geplante Forschungsvorhaben als wenig zielführend (vgl. dazu Kap. 2.4. bzw. MDa).

Die Veröffentlichung unzähliger NSA-Dokumente im Juni 2013 ging als Snowden Leaks in die Geschichte ein und markierten einen Wendepunkt in der Forschungslandschaft zu digitaler Überwachung. Auch für das vorliegende Forschungsvorhaben verschoben sich unter dem Eindruck dessen die Prioritäten und die Aspekte Privatsphäre und Datenschutz rückten in den Vordergrund. Ein Wechsel des universitären Standorts und entsprechend auch der Betreuung von Innsbruck nach Köln bedingte einen teilweisen Neuanfang, ein neues Exposé entstand und ein neues Forschungsprojekt nahm Konturen an. Die Mediennutzung junger Erwachsener in Bezug auf digitale Daten und ihre Privatsphäre sollte in einer ethnografisch angereicherten Interviewstudie untersucht werden.

Erneut kündigten sich Engpässe an - waren es keine finanziellen, so waren es zeitliche - und vice versa. Während all dieser Zeit, von 2012 bis 2017, lief das Tagesgeschäft weiter: Tagen und Vortragen, Lehren und Lernen, Lesen und Publizieren. Als die Selbstironie bezüglich der eigenen Dissertation ein kaum mehr steigerbares Mass erreichte, war gleichzeitig klar: Die Bedingungen erlauben es nicht, ein umfassendes Forschungsprojekt durchzuführen und eine Monografie zu verfassen. Glücklicherweise erlaubt es die Promotionsordnung der Humanwissenschaftlichen Fakultät an der Universität zu Köln, kumulative Dissertationen zu verfassen. Andernfalls wären die grossen Pläne womöglich vollends zum Scheitern verurteilt gewesen. In dieser kumulierten und theoretisch gehaltenen Form hingegen, so hoffe ich, können 
bestimmte Aspekte anders - und vielleicht sogar auf besonders interessante Weise ihre Darstellung finden, doch dazu mehr im kommenden Abschnitt «Zur (medialen) Form».

\section{Zur (medialen) Form}

Soweit mir bekannt ist, liegt hiermit die erste kumulative, also publikationsbasierte Dissertation im Fach Erziehungswissenschaft an der Universität zu Köln vor. Da das Prinzip einer kumulativen Dissertation in der Erziehungswissenschaft als umstritten und keineswegs als etabliertes Format bezeichnet werden kann, scheint es mir geboten, einige Worte über die Form der vorliegenden Dissertation zu verlieren.

Die an diesem Format für Qualifizierungsarbeiten wohl deutlichste Kritik im engeren Umfeld der Deutschen Gesellschaft für Erziehungswissenschaft (DGfE) hat Andreas Gruschka (2012) formuliert. Einigen Argumenten, die er gegen publikationsbasierte Dissertationen vorbringt, kann ich ganz oder teilweise zuzustimmen: Insbesondere betrifft das Vorteile für Promovend ${ }^{*} i n n e n^{1}$ in Forschungsgruppen und Interessenskonflikte durch Rollenüberschneidungen (z.B. als Betreuer ${ }^{\star}$ in und Co-Autor ${ }^{\star}$ in). Andere Argumente treffen m.E. auch auf monografisch verfasste Dissertationen ${ }^{2}$ in verwandter Form zu: Publizieren als Karriereinstrument, Eindeutigkeit von Autor*innenschaft, Knappheit hochwertiger Publikationsplätze, mögliche Vorteile durch soziales Kapital, Interessenskonflikte durch Rollenüberschneidungen (z.B. als Betreuer ${ }^{\star}$ in und Vorgesetzte* ${ }^{\star}$ ), Problematik der Abgrenzung der ceigenen Erkenntnisse> von anderen, kooperativen Arbeiten. Das Bild von kumulativen Dissertationen, das Gruschka zeichnet, lässt sich in etwa so skizzieren: Eine Person betreibt als wissenschaftliche ${ }^{\star} r$ Mitarbeiter ${ }^{\star}$ in in einer Forschungsgruppe arbeitsteilig organisierte, (vermutlich) empirische Bildungsforschung experimenteller Ausrichtung. Die Studien und Publikationen werden ausschliesslich kooperativ ersonnen, durchgeführt und verfasst - allerdings mit relativ stabilen (und womöglich hierarchischen) Rollenverteilungen.

1 In der gesamten Arbeit wird nach Möglichkeit konsequent diese Schreibung für geschlechtlich kodierte Ausdrücke benutzt. Ähnlich dem Gender-Gap (_) markiert der Asterisk $\iota^{\star}$ ) mögliche geschlechtliche Identitäten jenseits der heterosexuellen Matrix von Mann und Frau und der daran anschliessenden, als normal geltenden Begehrensformen. Vor dem Hintergrund der vorliegenden Arbeit kann dieses Vorgehen als ein medienkritisches aufgefasst werden, welches die symbolisch-medialen Rahmen der Wahrnehmung in Frage zu stellen versucht.

2 Nicht-publikationsbasierte, d.h. buchbasierte Dissertationen werden in der auf mich anzuwendenden Promotionsordnung als «monographische Dissertationen» bezeichnet. An diese Bezeichnung halte ich mich im Folgenden. Genau genommen ist allerdings unklar, ob mit dem vorliegenden Band nicht auch eine Monografie vorliegt, die nominell aus der Feder eines Autors stammt. Ohnehin ist eine solche Konzeption von Autor*in vor dem Hintergrund der Theoriebezüge in dieser Dissertation grundlegend in Frage zu stellen. Im Vorwort mögen diese Fussnote und ein pragmatisches Vorgehen der Problematik Genüge tun. 
Selbst wenn dieses Szenario prägend für kumulative Dissertationen sein mag; keines der Merkmale trifft auf die vorliegende zu. Der einzige Aspekt, der ihr offensichtlich problematisch werden kann, ist jener der nicht letztgültigen Unterscheidbarkeit der individuellen Beiträge in gemeinsam verfassten Texten. Abgesehen davon besteht dieses Problem - ob durch mehrere namentlich ausgewiesene Autor*innen gekennzeichnet oder nicht - für jeden wissenschaftlichen Text. Im Sinne einer poststrukturalistisch gedachten Intertextualität lassen sich weder das Konzept einer eindeutigen Urheber*innenschaft noch der Ursprünglichkeit von Sinnproduktion aufrecht erhalten. Im Gegenzug zum Beharren auf solchen Vorstellungen möchte ich die Potenziale kooperativer und kollaborativer wissenschaftlicher Arbeit betonen (freilich innerhalb ihrer Bedingtheiten und Grenzen). Umso mehr gilt dies, wenn die Kooperation nicht hierarchisch gegliedert ist, sondern in einer Weise, die über rein additive Produktion von Forschungsergebnissen, Erkenntnissen oder Texten hinausgeht und gerade dadurch, im besten Sinne, die Grenze zwischen «Einzelleistungen` verwischen muss.

In einer Arbeit, die sich auf medientheoretische Ansätze beruft, liegt die Reflexion über die eigene mediale Darstellungsform nahe. So muss etwa in Frage gestellt werden, ob es die mediale Form der Qualifizierungsarbeit ist (in diesem Fall: monografische oder kumulative Dissertation), aus der jene zu kritisierenden Aspekte entspringen - oder ob es vielmehr Kontextbedingungen wissenschaftlicher Tätigkeit sind, denen problematische Entwicklungen zuzuschreiben sind (vgl. dazu etwa Dander u.a. 2016). Sofern Wissenschaft auf Konkurrenz, Marktförmigkeit und künstlicher Verknappung basiert und vielfach unter Bedingungen fehlender Finanzierung stattfindet, werden sich sowohl die ungerechte Verteilung von knappem sozialem, kulturellem und ökonomischem Kapital, als auch um sich greifender Karrierismus in allen wissenschaftlichen Texten niederschlagen. Insofern ist die Form kumulativer Dissertationen in der Erziehungswissenschaft eher Symptom übergeordneter Tendenzen und weniger Ursache der Misere. Die notwendige Kritik muss daher an anderer Stelle ansetzen.

Jenseits solcher wissenschaftspolitischen Debatten möchte ich für meinen Einzelfall einige positive Aspekte einer kumulativen Dissertation nennen, um deutlich zu machen, dass das von Gruschka imaginierte Szenario, so zutreffend es in Tendenz sein mag, nicht als einzig mögliches zu begreifen ist. Eine Monografie bringt es trotz der langen Zeit des Schreibens mit sich, das Unmögliche zu behaupten: zu einem Zeitpunkt $X$ der Fertigstellung soll alles zuvor Geschriebene in sich aktuell und stimmig sein. Der Zeitpunkt der Veröffentlichung gilt für das gesamte Buch. Mehrere kleinere Texte in zeitlichen Abständen bringen mit sich, den Entwicklungsprozess (ich möchte sagen: Bildungsprozess), der Forschung auch immer ist, in der Arbeit in Kaskaden abzubilden und transparent zu machen (vgl. Prager 2013, 160). Frühere Beiträge dürfen als solche in das Konvolut eingehen, wodurch auf distanzierte Weise 
mit ihren Aussagen gearbeitet werden kann. Vielleicht etwas leichter als eine monografische Dissertation kann ein Konnex von Artikeln als Hypertextstruktur angelegt werden. Über die gesetzten Links im Text, die auf andere Texte verweisen, soll dieses Strukturmoment zumindest im Ansatz (und natürlich nur in der digitalen Version) zur Geltung kommen. Der vorliegende Manteltext bietet dabei lediglich eine aller möglichen Leseorientierungen.

Vielleicht wurden ältere Arbeiten bereits in der (akademischen) Öffentlichkeit diskutiert. Dieser Aspekt könnte als «Open Science ultralight` bezeichnet werden, insofern Zwischenschritte eines Promotionsprojekts öffentlich werden dürfen. Damit einher geht potenziell eine finanzielle Erleichterung, die - zumindest in einigen Fällen - mit dem Schlagwort Open Access zusammenhängt. Eine Monografie in einem Verlag zu publizieren, gilt als erstes Buch a als Pflichtübung für eine weitere wissenschaftliche Karriere. Der Druck (das Lektorat, der Satz, der Vertrieb, das (Freikaufen` von urheberrechtlichen Restriktionen etc.) ist mit Kosten verbunden. Sofern Zeitschriften-Redaktionen sich für eine Open Access-Politik entschieden haben - glücklicherweise ist das im medienpädagogischen Feld z.T. der Fall -, trifft das nicht in der Form zu. Ausserdem gewährleisten Open Access-Formate eine gewisse Unabhängigkeit von Verlagen sowie eine Reduktion der Zugangsbeschränkungen zu wissenschaftlichem Wissen für Andere, inner- wie ausserhalb des wissenschaftlichen Feldes. Open Science und Open Access sind nicht nur Gegenstände medienpädagogischer Forschung, sondern werden sinnvollerweise zum Strukturprinzip gemacht. Dass nicht jede Zeitschrift (Open Access oder nicht) gleichermassen hohe Qualitätsstandards ansetzt oder gewährleistet, mag zwar sein - doch das gilt nicht wesentlich anders für Buchpublikationen. Im Fall von begutachteten Zeitschriften-Artikeln tritt jedoch eine zusätzliche Ebene der fachlichen ‘Qualitätssicherung) hinzu.

Schliesslich bleibt die simple materielle Grundlage zu nennen, die auch für Forschung unumgänglich ist. Bereits im Rahmen einer Qualifikationsstelle für wissenschaftliche Mitarbeiter ${ }^{\star}$ innen oder einem Promotionsstipendium mit Laufzeiten von drei Jahren ist es kein einfaches, eine Dissertation in der gegebenen Zeit fertigzustellen. Immerhin gibt es aber eine Minimal-Sicherheit durch die Laufzeit der Stelle. Ist dies nicht gegeben, muss mit einer beständigen Unklarheit über die Finanzierung gearbeitet werden. In meinem Fall war es unter prekären Beschäftigungsverhältnissen mit wechselnden Standorten schlichtweg einfacher und adäquater, mehrere kürzere Arbeiten zu verfassen als ein Buch. Diese Situation ist kein Einzelfall, sondern trifft auf zahlreiche junge Forschende zu. ${ }^{3}$ Insofern bleibt zu hoffen, dass die genannten positiven Aspekte nicht nur in wenigen Einzelfällen zum Tragen kommen, sondern für möglichst viele einen angemesseneren Weg der Qualifikation eröffnen.

3 Vielleicht reichen zwei Zahlen als Beleg: 93\% des sogenannten wissenschaftlichen Nachwuchses an Hochschulen haben laut Bundesbericht Wissenschaftlicher Nachwuchs 2017 befristete Stellen. Etwas mehr als die Hälfte davon, so weisen es die einzig zugänglichen Zahlen aus, sieht sich dabei mit Vertragslaufzeiten von weniger als einem Jahr konfrontiert (vgl. Konsortium BuWIN 2017, $29 f$.). 
Die Gliederung der Arbeit wurde wie folgt aufgebaut: Nach dem «Manteltext` als inhaltliche Einführung und Leseorientierung folgen alle Teil-Publikationen nach dem Zeitpunkt des Erscheinens in einheitlichem Layout. Keine andere Ordnung wäre wesentlich schlüssiger gewesen, wie auch diese nicht in letzter Instanz schlüssig ist, da das 'Verfassen) und (Erscheinen` von Texten mitunter zeitlich stark voneinander abweicht. Die ausgewiesenen Seiten der Zitate im Manteltext orientieren sich, sofern bereits erschienen, an den jeweils publizierten Versionen. Im Text werden die Referenzen auf die einzelnen Teil-Publikationen durch Siglen ausgewiesen, zu denen sich unter «Siglen» eine Übersicht findet.

\section{Dank-Sagen}

Es wurde bereits gesagt: Es ist nicht zeitgemäss und es wäre angesichts der Argumentation der Arbeit alles andere als konsequent davon auszugehen, dass dieses Buch der Genialität des Verfassers geschuldet sei. Es ist es nicht und diese Genialität gibt es nicht. Unzählige Menschen und die Begegnungen mit ihnen sind Bedingungen für diese Arbeit. Ihre Zeit, ihr Vertrauen, ihre Klugheit und ihr kritisches Nachhaken, ihre Aufmunterung und Unterstützung und vieles mehr sind in den besten Teilen dieser Arbeit wiederzufinden. Dafür möchte ich Euch, die Ihr Euch jetzt zu Recht angesprochen fühlt, danke sagen: Danke! Für alle anderen Teile übernehme ich die Verantwortung im Rahmen der Möglichkeiten eines post-souveränen Subjekts.

Ebenfalls bedanke ich mich bei einem Fördergeber, der zeitweilig für die Finanzierung meiner Forschungsarbeit gesorgt hat. Im Rahmen des Nachwuchs-Programms der Leopold-Franzens-Universität Innsbruck bezog ich von April 2013 bis März 2014 ein Dissertationsstipendium, wodurch u.a. das Verfassen der Texte HGK, GvD, KRB und DuD massgeblich mit-ermöglicht wurde. 


\section{Siglen}

Die folgenden Aufsätze gehen als Teil-Publikationen in die vorliegende Dissertation ein und werden im Manteltext wie folgt referenziert:

\begin{tabular}{|c|c|}
\hline HGK & $\begin{array}{l}\text { How to Gain Knowledge When Data Are Shared? Open Government Data from a } \\
\text { Media Pedagogical Perspective (Dander 2014a) }\end{array}$ \\
\hline GvD & $\begin{array}{l}\text { Von der «Macht der Daten` zur «Gemachtheit von Daten». Praktische Datenkritik } \\
\text { als Gegenstand der Medienpädagogik (Dander 2014b) }\end{array}$ \\
\hline KRB & $\begin{array}{l}\text { Die Kunst des Reg(istr)ierens mit Big Data. Ein Versuch über Digitale Selbstvertei- } \\
\text { digung und Aktive Medienarbeit mit Daten (Dander 2014c) }\end{array}$ \\
\hline DuD & $\begin{array}{l}\text { Datendandyismus und Datenbildung. Von einer Rekonstruktion der Begriffe zu } \\
\text { Perspektiven der sinnvollen Nutzung (Dander 2014d) }\end{array}$ \\
\hline DPD & $\begin{array}{l}\text { Daten + Praxis = Datenhandeln? Eine Akzentverschiebung entlang der praxeologi- } \\
\text { schen Diskursanalyse (Dander 2015) }\end{array}$ \\
\hline MuD & $\begin{array}{l}\text { Medienpädagogik und (Big) Data: Konsequenzen für die erziehungswissenschaftli- } \\
\text { che Medienforschung und -praxis (Dander/Aßmann 2015) }\end{array}$ \\
\hline MDK & $\begin{array}{l}\text { Medien - Diskurs - Kritik. Potenziale der Diskursforschung für die Medienpädago- } \\
\text { gik (Dander 2017a) }\end{array}$ \\
\hline $\mathrm{MDa}$ & $\begin{array}{l}\text { Mediale Dispositivanalysen in der erziehungswissenschaftlichen Medienfor- } \\
\text { schung: Von Fallstricken und Auffangnetzen (Dander 2017b) }\end{array}$ \\
\hline WmM & $\begin{array}{l}\text { Wie «medienkritisch〉 ist die Medienpädagogik? Fragen und Angebote zu Analyse, } \\
\text { Ethik und Selbstreflexion einer «Disziplin〉 (Dander 2017c) }\end{array}$ \\
\hline STJ & $\begin{array}{l}\text { Self-Tracking als Gegenstand medienpädagogischer Jugendarbeit? (Dander } \\
\text { 2017d) }\end{array}$ \\
\hline $\mathrm{NzE}$ & $\begin{array}{l}\text { ¿das Netz, das zwischen diesen Elementen geknüpft werden kann〉. Theoretische } \\
\text { Verbindungslinien zwischen Netzwerk, Medien und Dispositiven (Dander 2018a) }\end{array}$ \\
\hline HTI & $\begin{array}{l}\text { Heterotopische Interventionen: Wissenskritische und bildungstheoretische Analy- } \\
\text { sen von Zone*Interdite und Poems from Guantánamo (Prager/Dander 2018b) }\end{array}$ \\
\hline KPÖ & $\begin{array}{l}\text { Grundzüge einer Kritischen Politischen Ökonomie von Big Data Analytics - und } \\
\text { ihre bildungstheoretischen Implikationen (Dander, i.E.) }\end{array}$ \\
\hline
\end{tabular}




\section{Literatur}

\section{Teil-Publikationen der kumulativen Dissertation}

Dander, Valentin. 2014a. «How to Gain Knowledge When Data Are Shared? Open Government Data from a Media Pedagogical Perspective». Seminar.Net 10 (1): 1-12. https://journals. hioa.no/index.php/seminar/article/view/2377. [HGK].

Dander, Valentin. 2014b. «Von der «Macht der Daten` zur «Gemachtheit von Daten〉. Praktische Datenkritik als Gegenstand der Medienpädagogik». Herausgegeben von Marcus Burkhardt und Sebastian Gießmann. Mediale Kontrolle unter Beobachtung, Nr. 3 Datenkritik. http:// www.medialekontrolle.de/ausgaben/3-12014-datenkritik/. [GvD].

Dander, Valentin. 2014c. «Die Kunst des Reg(istr)ierens mit Big Data. Ein Versuch über Digitale Selbstverteidigung und Aktive Medienarbeit mit Daten». medienimpulse. Beiträge zur Medienpädagogik 4 (Steuerung, Kontrolle, Disziplin/Medienpädagogische Perspektiven auf Medien und/der Überwachung): 1-13. http://medienimpulse.at/articles/view/739. [KRB].

Dander, Valentin. 2014d. «Datendandyismus und Datenbildung. Von einer Rekonstruktion der Begriffe zu Perspektiven sinnvoller Nutzung». In Datenflut und Informationskanäle, herausgegeben von Heike Ortner, Daniel Pfurtscheller, Michaela Rizzolli, und Andreas Wiesinger, 113-29. Interdisziplinäre Forschung. Innsbruck: innsbruck university press. http://books. openedition.org/iup/1265. [DuD].

Dander, Valentin. 2015. «Diskurse + Praktiken = Datenhandeln? Eine Akzentverschiebung entlang der praxeologischen Diskursanalyse». medienimpulse. Beiträge zur Medienpädagogik 3 (Handeln mit Symbolen): 1-10. http://www.medienimpulse.at/articles/view/849. [DPD].

Aßmann, Sandra, und Valentin Dander. 2015. «Medienpädagogik und (Big) Data: Konsequenzen für die erziehungswissenschaftliche Medienforschung und -praxis». In Big Data und Medienbildung. Zwischen Kontrollverlust, Selbstverteidigung und Souveränität in der digitalen Welt, herausgegeben von Harald Gapski, 33-50. Schriftenreihe zur digitalen Gesellschaft NRW 3. Düsseldorf; München: kopaed. http://nbn-resolving.de/urn:nbn:de:0111pedocs-116340. [MuD].

Dander, Valentin. 2017a. «Medien - Diskurs - Kritik. Potenziale der Diskursforschung für die Medienpädagogik». Herausgegeben von Sven Kommer, Thorsten Junge, und Christiane Rust. MedienPädagogik: Zeitschrift für Theorie und Praxis der Medienbildung 27 (Tagungsband: Spannungsfelder und blinde Flecken. Medienpädagogik zwischen Emanzipationsanspruch und Diskursvermeidung): 50-64. https://doi.org/10.21240/mpaed/27/2017.01.15.X. [MDK].

Dander, Valentin. 2017b. «Mediale Dispositivanalysen in der erziehungswissenschaftlichen Medienforschung: Von Fallstricken und Auffangnetzen». In Forschungswerkstatt Medienpädagogik. Projekt - Theorie - Methode, herausgegeben von Thomas Knaus, 1-20. München: kopaed. http://publ.forschungswerkstatt-medienpaedagogik.de/download/\%2316873_Fo rschungswerkstattMedienp\%C3\%A4dagogik_Dander_Entw22_17Jul05.pdf. [MDa]. 
Dander, Valentin. 2017c. «Wie «medienkritisch» ist Medienpädagogik? Fragen und mögliche Antworten zu Analyse, Ethik und Selbstreflexion einer ¿Disziplin»». Herausgegeben von Christian Swertz, Wolfgang B. Ruge, Alexander Schmölz, Alessandro Barberi, und Sarah Braun. MedienPädagogik: Zeitschrift für Theorie und Praxis der Medienbildung 29 (Die Konstitution der Medienpädagogik. Zwischen interdisziplinärem Forschungsfeld und bildungswissenschaftlicher (Sub-) Disziplin): 105-38. https://doi.org/10.21240/mpaed/29/2017.09.05.X. [WmM].

Dander, Valentin. 2017d. «Self-Tracking als Gegenstand medienpädagogischer Jugendarbeit?» merz - medien+erziehung 5 (Self-Tracking. Lifelogging. Quantified Self). [STJ].

Dander, Valentin. 2018. ««Das Netz, das zwischen diesen Elementen geknüpft werden kann». Theoretische Verbindungslinien zwischen Netzwerk, Medien und Dispositiven». In Netzwerk als neues Paradigma?, herausgegeben von Florian Kiefer und Jens Holze, 65-81. Medienbildung und Gesellschaft 39. Wiesbaden: Springer Fachmedien Wiesbaden. https:// doi.org/10.1007/978-3-658-18003-4_4. [NzE].

Prager, Julia, und Valentin Dander. 2018. «Heterotopische Interventionen Wissenskritische und bildungstheoretische Analysen von Zone*Interdite und Poems from Guantánamo». In Verkörperte Heterotopien. Zur Materialität und [Un-]Ordnung ganz anderer Räume, herausgegeben von Lea Spahn, Jasmin Scholle, Bettina Wuttig, und Susanne Maurer. Bielefeld: transcript Verlag. https://doi.org/10.14361/9783839438732-019. [HTI].

Dander, Valentin. im Erscheinen. "Grundzüge einer Kritischen Politischen Ökonomie von Big Data Analytics - und ihre bildungstheoretischen Implikationen». In Big Data (Proceedings zum Magdeburger Theorieforum 2016). Wiesbaden: VS Verlag für Sozialwissenschaften. [KPÖ]. 